University of Warwick institutional repository: http://go.warwick.ac.uk/wrap This paper is made available online in accordance with publisher policies. Please scroll down to view the document itself. Please refer to the repository record for this item and our policy information available from the repository home page for further information.

To see the final version of this paper please visit the publisher's website. Access to the published version may require a subscription.

\author{
Author(s): IAN STEWART \\ Article Title: The lattice of balanced equivalence relations of a coupled \\ cell network \\ Year of publication: 2007 \\ Link to published version: http://dx.doi.org/ \\ 10.1017/S0305004107000345 \\ Publisher statement: None
}




\title{
The lattice of balanced equivalence relations of a coupled cell network
}

\author{
BY IAN STEWART \\ Mathematics Institute, University of Warwick, Coventry CV4 7AL.
}

(Received 26 April 2006; revised 15 August 2006)

\begin{abstract}
A coupled cell system is a collection of dynamical systems, or 'cells', that are coupled together. The associated coupled cell network is a labelled directed graph that indicates how the cells are coupled, and which cells are equivalent. Golubitsky, Stewart, Pivato and Török have presented a framework for coupled cell systems that permits a classification of robust synchrony in terms of the concept of a 'balanced equivalence relation', which depends solely on the network architecture. In their approach the network is assumed to be finite. We prove that the set of all balanced equivalence relations on a network forms a lattice, in the sense of a partially ordered set in which any two elements have a meet and a join. The partial order is defined by refinement. Some aspects of the theory make use of infinite networks, so we work in the category of networks of 'finite type', a class that includes all locally finite networks. This context requires some modifications to the standard framework. As partial compensation, the lattice of balanced equivalence relations can then be proved complete. However, the intersection of two balanced equivalence relations need not be balanced, as we show by a simple example, so this lattice is not a sublattice of the lattice of all equivalence relations with its usual operations of meet and join. We discuss the structure of this lattice and computational issues associated with it. In particular, we describe how to determine whether the lattice contains more than the equality relation. As an example, we derive the form of the lattice for a linear chain of identical cells with feedback.
\end{abstract}

\section{Introduction}

The dynamics of networks (of coupled systems of differential equations) is a topic of considerable current activity (Manrubia et al. [9], Mosekilde et al. [10], Wu [13, 14]). The topic has intrinsic mathematical interest, and arises in numerous areas of applied science, for example the Internet, the spread of epidemics, food webs in ecosystems, neural circuits, gene transcription and cellular signalling. The literature is too large to cite specific articles, but see the survey [5] for a sample.

We will work in a formal framework for the dynamics of networks, based on the 'symmetry groupoid' of the network, introduced in [11]. This formalism was improved by Golubitsky et al. [6] to permit multiple edges and loops connecting a given node to itself. In both versions of the formalism, the nodes ('cells') and directed edges ('arrows') are classified into various types, and the symmetry groupoid consists of all type-preserving isomorphisms of 'input sets' - those arrows whose heads are a given cell, along with the tails of those arrows. The symmetry groupoid encodes the constraints imposed on the dynamics by 
the requirement that certain cells or arrows have the same type. This 'coupled cell network' formalism is surveyed in [5].

An important feature of networks is the possibility of synchrony, which occurs when distinct nodes exhibit identical dynamics at all instants of time. The coupled cell formalism is especially suited to the characterisation of patterns of synchrony. Indeed, a basic theorem ([11, theorem 6.5]) states that all 'robust' patterns of synchrony-those determined by the network architecture rather than any specific choice of dynamic - correspond to 'balanced equivalence relations' on the set of cells. This combinatorial condition formalises the property 'synchronous' as an equivalence relation on cells. Roughly speaking, balance means that synchronous cells must have synchronous input sets, up to a type-preserving isomorphism.

It therefore becomes of interest to determine all possible balanced equivalence relations on a given network. In general there may be many such relations, so it is reasonable to ask what structure the set of all balanced equivalence relations possesses. Here we show that for a broad class of networks, which includes all finite and locally finite networks, this set constitutes a complete lattice in the sense of Davey and Priestley [3]. It is well known that the set of all equivalence relations on a given set also forms a complete lattice, with the 'meet' operation being intersection. However, we show in Example 5.5 that the intersection of two balanced equivalence relations need not be balanced. In particular, the set of balanced equivalence relations is not a complete sublattice of the lattice of all equivalence relations. However, these two lattices share the same join operation.

This fact complicates the description of the lattice operations for balanced equivalence relations, and indicates that a different approach is needed. The complete lattice property of balanced equivalence relations seems to follow most naturally from a connection between balanced equivalence relations and flow-invariant subspaces.

One consequence is that on every network there exists a unique coarsest balanced equivalence relation. Here, the number of synchronous sets of cells is as small as possible. One way to decide whether a given network possesses any nontrivial balanced equivalence relations (that is, distinct from the relation of equality, which corresponds to no synchrony relations between distinct cells) is to compute this coarsest relation. Aldis [1] has found an algorithm to compute the coarsest balanced equivalence relation in polynomial time (in the number $k$ of cells plus the number of edges). Indeed, the time is bounded by a constant multiple of the fourth power of $k$, and seems to be entirely practical. His algorithm is based in part on the results of this paper.

\subsection{Structure of paper}

In Section 2 we recall the formal definition of a coupled cell network in the multiarrow version, and introduce the class of networks of finite type. Section 3 introduces the space of admissible maps on a network and proves that for networks of finite type the flow-invariant polydiagonals are precisely those that correspond to balanced equivalence relations. Section 4 summarises the properties of complete lattices that we require.

The main result of the paper, in Section 5 is a proof that on any network of finite type the set of balanced equivalence relations has the natural structure of a complete lattice. The proof exploits the relation between balanced equivalence relations and flow-invariant polydiagonals, which is an order-reversing bijection. We also give the aforementioned example of two balanced equivalence relations whose intersection is not balanced.

In Section 6 we discuss the coarsest balanced equivalence relation on a network, and in Section 7 we characterise this relation in terms of 'input trees' of cells. Here we use 


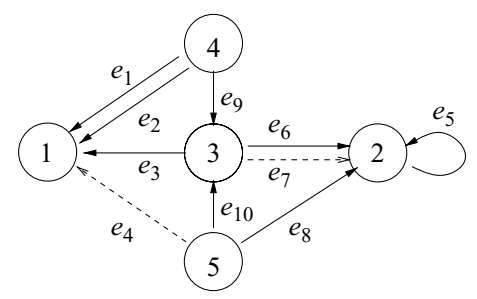

Fig. 1. Example of coupled cell network.

the universal cover of the network, which in general is a forest. Finally, in Section 8, we work out the lattice of balanced equivalence relations of a class of networks with simple but significant topology, linear chains with feedback.

\section{Coupled cell networks}

To make this paper self-contained we briefly recall the basic definitions and properties of coupled cell networks in the multiarrow formalism. For motivation and proofs, see [5, 6, 11]. Some existing proofs assume that the network is finite, so we rework these proofs in the more general context assumed in this paper.

\subsection{Architecture}

Definition 2.1. A coupled cell network $\mathcal{G}$ comprises:

(a) A set $\mathcal{C}$ of nodes or cells.

For applications to dynamics, this set is usually taken to be finite, with $\mathcal{C}=$ $\{1, \ldots, N\}$ for a positive integer $N$. Because certain infinite graphs arise in this paper, we will impose no such restriction on $\mathcal{C}$.

(b) An equivalence relation $\sim_{C}$ on cells in $\mathcal{C}$, called cell-equivalence.

The type or cell label of a cell is its $\sim_{C}$-equivalence class.

(c) A set $\mathcal{E}$ of edges or arrows.

Again, $\mathcal{E}$ is usually assumed to be finite, but we impose no such restriction here.

(d) An equivalence relation $\sim_{E}$ on edges in $\mathcal{E}$, called edge-equivalence or arrow-equivalence.

The type or coupling label of an arrow is its $\sim_{E}$-equivalence class.

(e) Two maps $\mathcal{H}: \mathcal{E} \rightarrow \mathcal{C}$ and $\mathcal{T}: \mathcal{E} \rightarrow \mathcal{C}$.

For $e \in \mathcal{E}$ we call $\mathcal{H}(e)$ the head of $e$ and $\mathcal{T}(e)$ the tail of $e$, which are required to satisfy the following consistency condition: Equivalent arrows have equivalent tails and heads. That is, if $e_{1}, e_{2} \in \mathcal{E}$ and $e_{1} \sim_{E} e_{2}$, then

$$
\mathcal{H}\left(e_{1}\right) \sim_{C} \mathcal{H}\left(e_{2}\right) \quad \mathcal{T}\left(e_{1}\right) \sim_{C} \mathcal{T}\left(e_{2}\right) .
$$

In this definition, an arrow may be a self-connection (that is, $\mathcal{T}(e)$ may be the same as $\mathcal{H}(e))$ and multiple arrows may exist (distinct arrows $e, e^{\prime}$ may have $\mathcal{T}(e)=\mathcal{T}\left(e^{\prime}\right)$ and $\mathcal{H}(e)=$ $\left.\mathcal{H}\left(e^{\prime}\right)\right)$. However, the multiplicity of each arrow is always finite.

\section{Example 2.2.}

The coupled cell network in Figure 1 has five cells, so $\mathcal{C}=\{1,2,3,4,5\}$, and ten edges, so $\mathcal{E}=\left\{e_{1}, \ldots, e_{10}\right\}$. The cells are all $\sim_{C}$-equivalent, shown by using the same symbol (circle) for each of them. There are two arrow-types: the solid arrows $\left\{e_{1}, e_{2}, e_{3}, e_{5}, e_{6}, e_{8}\right.$, $\left.e_{9}, e_{10}\right\}$ and the dotted arrows $\left\{e_{4}, e_{7}\right\}$. There is a double arrow, comprising $e_{1}, e_{2}$, from cell 4 to cell 1 , and a self-connection $e_{5}$ at cell 2 . 

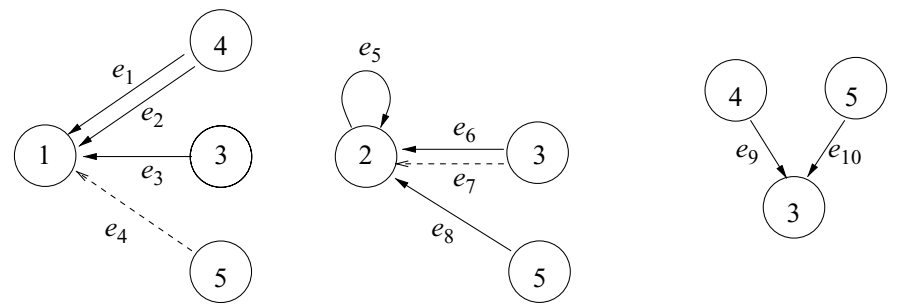

Fig. 2. Input sets in the network of Figure 1. (Left) $I$ (1). (Middle) $I(2)$. (Right) $I(3)$.

This example has no special significance, but is chosen to exhibit a few key concepts in a simple manner.

Associated with each cell $c \in \mathcal{C}$ is a canonical set of edges, namely, those that represent couplings into cell $c$ :

Definition 2.3. If $c \in \mathcal{C}$ then the input set of $c$ is

$$
I(c)=\{e \in \mathcal{E}: \mathcal{H}(e)=c\} .
$$

An element of $I(c)$ is called an input edge or input arrow of $c$.

Remark 2.4. A cell may have empty input set (consider cells 4 and 5 in Figure 1). This possibility does not arise in the 'single arrow' formalism of [11]. It leads to some slightly pedantic considerations about mappings involving the empty set: see Remarks 2.7 and $5 \cdot 2$ below.

Important features of a network are captured by an equivalence relation that is determined by the structure of input sets:

Definition 2.5. The relation $\sim_{I}$ of input equivalence on $\mathcal{C}$ is defined by $c \sim_{I} d$ if and only if there exists a bijection

$$
\beta: I(c) \longrightarrow I(d)
$$

such that for every $i \in I(c)$,

$$
i \sim_{E} \beta(i)
$$

Any such bijection $\beta$ is called an input isomorphism from cell $c$ to cell $d$.

\section{Example 2.6.}

The input sets $I(1), I(2)$, and $I(3)$ for Figure 1 are shown in Figure 2. The other two input sets are empty. For clarity, the figure shows the head and tail cells as well as the input arrows. Cells 1 and 2 are input-isomorphic; one suitable $\beta$ is

$$
\left(\begin{array}{llll}
e_{1} & e_{2} & e_{3} & e_{4} \\
e_{5} & e_{6} & e_{8} & e_{7}
\end{array}\right)
$$

in 'partial permutation' notation, where the bottom row is the result of applying $\beta$ to the top row. There are five other possibilities for $\beta$, obtained by permuting $e_{5}, e_{6}, e_{8}$. In contrast, cell 3 is not input-isomorphic to cell 1 .

Remark 2.7. If $I(c)=I(d)=\varnothing$, then the set $B(c, d)$ is not empty. With the standard definition of a function $f: A \rightarrow B$ as a subset of $A \times B$ with certain properties, there exists a unique function $f: \varnothing \rightarrow \varnothing$. Indeed, $f$ is itself the empty set. This $f$ is a bijection for trivial reasons. The set $B(c, d)$ is then equal to $\{\varnothing\}$. 


\section{Lattice of balanced equivalence relations}

It will prove useful to distinguish the input arrows for a given cell $c \in \mathcal{C}$ according to their type:

Definition 2.8. Let $\mathcal{G}$ be a network, let $c \in \mathcal{C}$ and let $A$ denote an arrow-type of $\mathcal{G}$, that is, $\mathrm{a} \sim_{E}$-equivalence class. Define

$$
I^{A}(c)=\{e \in I(c): e \in A\}=I(c) \cap A .
$$

We use the notation $\mathcal{A}_{\mathcal{G}}$ for the set of all arrow-types of $\mathcal{G}$, and omit $\mathcal{G}$ when the network concerned is obvious.

We now impose a finiteness condition on the network, which seems to be the most general condition that extends the theory of finite networks. We motivate this definition in Section 3.

Definition 2.9. A coupled cell network $\mathcal{G}$ is of finite type if $I^{A}(c)$ is finite for all $c \in$ $\mathcal{C}, A \in \mathcal{A}_{\mathcal{G}}$.

Examples $2 \cdot 10$.

Every finite network is of finite type.

Every locally finite network (that is, one for which $I(c)$ is finite for all $c \in \mathcal{C}$ ) is of finite type.

Let $\mathcal{C}=\mathbf{Z}^{2}$ be the two-dimensional square lattice. The symmetry group of this lattice is $\Gamma=\left(\mathbf{Z}_{2} \oplus \mathbf{Z}_{2}\right) \dot{+} \mathbf{D}_{4}$ where $\mathbf{Z}_{2} \oplus \mathbf{Z}_{2}$ acts by translations and $\mathbf{D}_{4}$ is the holohedry, see [2]. As defined in that paper, the group network corresponding to $\Gamma$ acting on $\mathcal{C}$ has a unique arrow $e_{c d}$ for all $c \neq d \in \mathcal{C}$, with $\mathcal{T}\left(e_{c d}\right)=c, \mathcal{H}\left(e_{c d}\right)=d$. The arrow-type is determined by the $\Gamma$-action, so that $e_{c, d} \sim_{E} e_{c^{\prime}, d^{\prime}}$ if and only if there exists $\gamma \in \Gamma$ such that $c^{\prime}=\gamma(c)$ and $d^{\prime}=\gamma(d)$. The resulting network $\mathcal{G}_{\Gamma}$ is of finite type, because it is easy to verify that $\left|I^{A}(c)\right| \leqslant 8$ for all $c, A$. It is not locally finite, because $I(c)$ involves infinitely many distinct arrow-types.

Definition $2 \cdot 11$. The set $B(c, d)$ denotes the collection of all input isomorphisms from cell $c$ to cell $d$. The union

$$
\mathcal{B}_{\mathcal{G}}=\bigcup_{c, d \in \mathcal{C}} B(c, d)
$$

is the symmetry groupoid of the network $\mathcal{G}$. It is a groupoid in the sense of Higgins [8].

For any $c \in \mathcal{C}$, the set $B(c, c)$ is a group, called the vertex group at $c$.

\section{Admissible maps}

We now define admissible maps, for a given coupled cell network $\mathcal{G}$, which are maps that are consistent with the labelled graph structure, or equivalently are compatible with, and symmetric under, the groupoid $\mathcal{B}_{\mathcal{G}}$.

For each cell $c \in \mathcal{C}$ we first choose a cell phase space $P_{c}$, which we here assume to be a nonzero finite-dimensional real vector space. In order to compare distinct cells we require equivalent cells to have the same (canonically identified) phase spaces:

$$
c \sim_{C} d \Longrightarrow P_{c}=P_{d} .
$$

We then employ the same coordinate systems on $P_{c}$ and $P_{d}$. The total phase space of $\mathcal{G}$ is the cartesian product

$$
P=\prod_{c \in \mathcal{C}} P_{c}
$$


with a cell-based coordinate system

$$
x=\left(x_{c}\right)_{c \in \mathcal{C}} .
$$

If $\mathcal{D} \subseteq \mathcal{C}$ then we write

$$
P_{\mathcal{D}}=\prod_{d \in \mathcal{D}} P_{d} .
$$

For any $\beta \in B(c, d)$ we define the pullback map

$$
\beta^{*}: P_{\mathcal{T}_{(I(d))}} \longrightarrow P_{\mathcal{T}_{(I(c))}}
$$

by

$$
\left(\beta^{*} z\right)_{\mathcal{T}_{(i)}}=z_{\mathcal{T}_{(\beta(i))}}
$$

for all $i \in I(c)$ and $z \in P_{\mathcal{T}_{(I(d))}}$.

We now introduce a key concept:

Definition 3.1. A map $f: P \rightarrow P$ is $\mathcal{G}$-admissible if:

(a) Strong domain condition: for all $c \in \mathcal{C}$ the component $f_{c}(x)$ depends only on a finite subset of the internal phase space variables $x_{c}$ and the coupling phase space variables $x_{\mathcal{T}_{(I(c))}}$. Specifically, for each $c \in \mathcal{C}$ there exists a finite union $\mathbf{A}=A_{1} \cup \ldots \cup A_{s}$ of arrow-types, which may depend on $c$, and a linear map

$$
\hat{f}_{c}: P_{c} \times P_{\mathcal{T}_{(I(c) \cap \mathbf{A})}} \longrightarrow P_{c}
$$

such that

$$
f_{c}(x)=\hat{f}_{c}\left(x_{c}, x_{\left.\mathcal{T}_{(I(c) \cap \mathbf{A})}\right)} .\right.
$$

(b) Pullback condition: for all $c, d \in \mathcal{C}$ and $\beta \in B(c, d)$, and the above choice of $\mathbf{A}$,

$$
\hat{f}_{d}\left(x_{d}, x_{\mathcal{T}_{(I(d) \cap \mathbf{A})}}\right)=\hat{f}_{c}\left(x_{d}, \beta^{*} x_{\mathcal{T}_{(I(d) \cap \mathbf{A})}}\right)
$$

for all $x \in P$.

\section{Remarks 3.2.}

(a) Clearly $I(d) \cap \mathbf{A}=I^{A_{1}}(d) \cup \ldots \cup I^{A_{s}}(d)$.

(b) The strong domain condition, applied to a network of finite type, does not in general reduce the analysis to locally finite networks, because the number of arrow-types occurring in $\mathbf{A}$ is not bounded as $c$ varies.

(c) In the above definition we have not specified any further structure on the map $f$. For connections with ODEs we require $f$ to have some degree of smoothness. For the purposes of this paper, linear maps suffice. In between are algebraic (polynomial) and analytic maps. When such properties are relevant, we say that $f$ is an admissible linear (or algebraic or analytic or smooth) map if and only if $\hat{f}_{c}$ satisfies the strong domain and pullback conditions, and is linear (respectively algebraic, analytic, smooth).

The strong domain condition (b) implies the usual domain condition for admissible vector

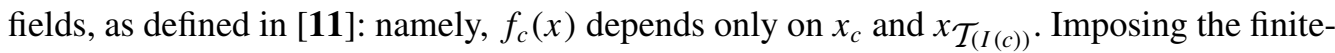
ness condition (3.2) lets us extend the theory to networks of finite type. Indeed, with this assumption we can characterise the admissible maps, in a manner that is analogous to [11, proposition 4.6]. We can also give a complete description of the admissible linear maps, which we will use in Theorem 3.7 to link balanced equivalence relations and admissibly invariant polydiagonals. 
Example 3.3.

To motivate the analysis we begin with an example: the group network $\mathcal{G}_{\mathbf{D}_{\infty}}$ of the infinite dihedral group $\mathbf{D}_{\infty}$ acting on the one-dimensional lattice $\mathbf{Z}$. Here the cells correspond to the integers $(c \in \mathbf{Z})$ and $\mathbf{D}_{\infty}$ is generated by the permutations $\sigma, \kappa$ where $\sigma(z)=z+1, \kappa(z)=$ $-z$ for $z \in \mathbf{Z}$. The arrow-types $A_{i}$ correspond to integers $i \geqslant 1$. For each $c \in \mathbf{Z}$ and $i>1$ there are two arrows $e_{c, i}^{ \pm}$of type $A_{i}$ such that

$$
\begin{array}{cc}
\mathcal{H}\left(e_{c, i}^{-}\right)=c & \mathcal{T}\left(e_{c, i}^{-}\right)=c-i, \\
\mathcal{H}\left(e_{c, i}^{+}\right)=c & \mathcal{T}\left(e_{c, i}^{+}\right)=c+i .
\end{array}
$$

For simplicity take $P_{c}=\mathbf{R}$ so that $P=\bigoplus_{c \in \mathbf{Z}} \mathbf{R}$. The vertex group $B(c, c)$ has order 2 , generated by the transposition that interchanges arrows $e_{c, i}^{+}$and $e_{c, i}^{-}$.

The most obvious $B(0,0)$-invariant linear maps $f_{0}: P \rightarrow \mathbf{R}$ are those of the form $f_{0}^{i}$, where

$$
f_{0}^{i}(x)=x_{i}+x_{-i}
$$

and it is easy to verify that these span the space of all $B(0,0)$-invariant linear maps satisfying the strong domain condition. By pullback, the corresponding admissible maps $f^{i}$ satisfy

$$
f_{c}^{i}(x)=x_{c+i}+x_{c-i} .
$$

There is one such map for each arrow-type, and they span the space of all admissible linear maps.

The classification of admissible linear maps for any network of finite type follows much the same pattern. To state and prove the classification, we need some notation. Recall the definition of $I^{A}(c)$ from Definition 2.8. Let $c \in \mathcal{C}$ and let $I(c)$ be its input set. (If there is a self-loop from $c$ to $c$, then the cell $c$ is a member of $\mathcal{T}(I(c))$ and the corresponding arrow must therefore lie in some $I^{A}(c)$.) Let $\mathcal{A}$ be the set of all arrow-types. Then

$$
I(c)=\bigcup_{A \in \mathcal{A}} I^{A}(c)
$$

and each set $I^{A}(c)$ is finite since $\mathcal{G}$ is of finite type. Define the multiplicity of arrow-type $A$ at $c$ to be

$$
n_{A}(c)=\left|I^{A}(c)\right| .
$$

The following lemma is then obvious from the definition of input isomorphism:

LEMMA 3.4. With the above notation, the vertex group

$$
B(c, c)=\prod_{A \in \mathcal{A}} \mathbf{S}_{n_{A}(c)}
$$

where $\mathbf{S}_{n_{A}(c)}$ is the symmetric group comprising all permutations of $I^{A}(c)$.

Note that each factor $\mathbf{S}_{n_{A}(c)}$ is finite, but there may be infinitely many factors (from the definition of 'finite type').

Let $\mathcal{R}$ be a set of representatives in $\mathcal{C}$ for input-equivalence, so that each cell $c \in \mathcal{C}$ is $\sim_{I}$-equivalent to exactly one $r \in \mathcal{R}$. It is possible to characterise all admissible maps $f$ in terms of the groups $B(r, r)$ (for $r \in \mathcal{R}$ ) and the pullback maps $\beta^{*}: I(d) \rightarrow I(r)$ for each $d \neq r$ such that $d \sim_{I} r$. Indeed, $f_{r}$ must be invariant under the action of $B(r, r)$, and then all other components are uniquely determined by pullback. The argument is exactly as in 
[11, lemma 4.5 and proposition 4.6], because the strong domain condition is consistent with pullback, and from any given invariant the same admissible map arises for any choice of pullback maps, by the usual argument [11]. We therefore have:

Proposition 3.5. A map $f: P \rightarrow P$ satisfying the strong domain condition is admissible if and only if:

(a) $f_{r}$ is invariant under $B(r, r)$;

(b) $f_{d}(x)=f_{r}\left(\beta^{*} x\right)$ for some, hence any, choice of $\beta: I(r) \rightarrow I(d)$.

It follows that we can characterise the admissible maps by finding the $B(r, r)$-invariant maps. But these are determined by Lemma 3.4. (Note that properties such as smoothness or linearity are preserved in this construction. In particular, $f$ is linear if and only if each $f_{r}$ is linear.)

\subsection{Averaging over the group}

It remains to determine the $B(r, r)$-invariant maps. To do so we recall a well-known technique from representation theory, see for example Fulton and Harris [4, proposition 2.8].

Suppose that a finite group $\Gamma$ acts linearly on a finite-dimensional vector space $X$, and let $Z$ be a finite-dimensional vector space. Suppose that $f: X \rightarrow Z$ is a linear map. Then we define the average of $f$ over the group $\Gamma$ to be the map $\bar{f}$ for which

$$
\bar{f}(x)=\frac{1}{|\Gamma|} \sum_{\gamma \in \Gamma} f(\gamma x) .
$$

It is easy to prove that $\bar{f}$ is $\Gamma$-invariant, in the sense that

$$
\bar{f}(\gamma x)=\bar{f}(x)
$$

for all $x \in X, \gamma \in \Gamma$. Moreover, if $f$ is $\Gamma$-invariant then $\bar{f}=f$. So averaging is a projection from the space of all maps onto the space of invariant maps.

We can apply this construction to the identity map on $X$, leading to the following notation:

$$
\bar{x}=\frac{1}{|\Gamma|} \sum_{\gamma \in \Gamma} \gamma x .
$$

If $f$ is linear, then (3.4) implies that

$$
\bar{f}(x)=f(\bar{x}) .
$$

This fact, though trivial, is very useful.

We pursue this connection in the linear case. We begin with a simple but useful lemma:

Lemma 3.6. Let $X, Y, Z$ be vector spaces, with $Y$ a subspace of $X$, and let a finite group $\Gamma$ act linearly on $X$. Suppose that a linear map $h: X \rightarrow Z$ is $\Gamma$-invariant when restricted to $Y$, in the sense that

$$
h(\gamma y)=F(y) \quad \forall y \in Y, \gamma \in \Gamma .
$$

Then there exists a $\Gamma$-invariant linear map $k: X \rightarrow Z$ such that $\left.k\right|_{Y}=\left.h\right|_{Y}$.

Proof. Define $k=\bar{h}$. Then $k$ is $\Gamma$-invariant and $\left.k\right|_{Y}=\left.h\right|_{Y}$ since $h$ is $\Gamma$-invariant when restricted to $Y$. 


\subsection{Action of the symmetric group}

The standard representation of the symmetric group $\mathbf{S}_{m}$ is its action on $\mathbf{R}^{m}$ by permutation of coordinates. If a finite-dimensional representation $X$ of $\mathbf{S}_{m}$ is a direct sum of copies of the standard representation, we say that $\mathbf{S}_{m}$ acts on $X$ by permutation. (We here use the term 'representation' to refer to what more pedantically would be called the 'representation space', as is usual.)

It is well known that if $\mathbf{S}_{m}$ acts on $X$ by permutation, then the invariant linear maps $f: X \rightarrow \mathbf{R}$ are precisely the linear maps of the form

$$
f(x)=g(\bar{x})
$$

where $g$ is any linear map $g: X \rightarrow \mathbf{R}$. (Proof: by invariance $f(x)=\bar{f}(x)$, but this equals $f(\bar{x})$. The converse is equally trivial.)

Now suppose that a finite collection of symmetric groups $\mathbf{S}_{m_{i}}$ act on spaces $X_{i}$ by permutation, where $1 \leqslant i \leqslant p$, and consider the diagonal action of $\mathbf{S}=\mathbf{S}_{m_{1}} \times \cdots \times \mathbf{S}_{m_{p}}$ on $Y=X_{1} \oplus \cdots \oplus X_{p}$ defined by

$$
\left(\gamma_{1}, \ldots, \gamma_{p}\right)\left(x_{1}, \ldots, x_{p}\right)=\left(\gamma_{1} x_{1}, \ldots, \gamma_{p} x_{p}\right) .
$$

Then every linear map $L: Y \rightarrow \mathbf{R}$ is uniquely of the form

$$
L(x)=L_{1}\left(x_{1}\right)+\cdots+L_{p}\left(x_{p}\right)
$$

for linear maps $L_{i}: X_{i} \rightarrow \mathbf{R}$. Clearly $L$ is $\mathbf{S}$-invariant if and only if each $L_{i}$ is $\mathbf{S}_{m_{i}}$-invariant. Therefore, writing

$$
\bar{x}_{i}=\frac{1}{\left|\mathbf{S}_{n_{i}}\right|} \sum_{\gamma \in \mathbf{S}_{n_{i}}} \gamma x_{i}
$$

we have

$$
L_{i}\left(x_{i}\right)=M_{i}\left(\bar{x}_{i}\right)
$$

for linear maps $M_{i}: X_{i} \rightarrow \mathbf{R}$. Therefore

$$
L(x)=M_{1}\left(\bar{x}_{1}\right)+\cdots+M_{p}\left(\bar{x}_{p}\right) .
$$

Now let $A$ be a fixed arrow-type, and for each $x \in P$ and $c \in \mathcal{C}$ define

$$
\bar{x}^{A, c}=\sum_{e \in A \cap I(c)} x_{\mathcal{T}_{(e)}} .
$$

Observe that all cells $\mathcal{T}(e)$ in this sum are cell-equivalent since $A$ is an arrow-type, so the sum makes sense because all phase spaces $P_{\mathcal{T}_{(e)}}$ are canonically identified. We now have:

THEOREM 3.7. Let $\mathcal{R}$ be a set of representatives for $\sim_{I}$. Every admissible linear map $f: P \rightarrow P$ can be uniquely expressed, via pullback, in terms of the components

$$
f_{r}(x)=M_{0}\left(x_{r}\right)+\sum_{A \in \mathbf{A}} M_{A}\left(\bar{x}^{A, r}\right) \quad(r \in \mathcal{R})
$$

for a finite set $\mathbf{A}$ of arrow-types. Here $M_{0}$ is any linear map $M_{0}: P_{c} \rightarrow P_{c}$, and for each $A \in$ A the map $M_{A}$ is any linear map $M_{A}: P_{j} \rightarrow P_{c}$ for $j \in A \cap I(c)$. (We make the convention that $M_{A}=0$ whenever $A \cap I(c)=\varnothing$.)

Proof. This is a restatement in coupled cell network notation of (3.7). 


\section{Complete lattices}

Next, we recall some basic facts about complete lattices. For further information see Davey and Priestley [3].

A lattice is a partially ordered set $X$ such that any two elements $x, y \in X$ have a unique greatest lower bound or meet, and a unique least upper bound or join, denoted respectively by

$$
x \wedge y \quad x \vee y .
$$

A lattice $X$ is complete if every subset $Y \subseteq X$ has a unique greatest lower bound or meet, and a unique least upper bound or join, denoted respectively by

$$
\bigwedge Y \quad \bigvee Y .
$$

Here $Y=\varnothing$ is allowed, so $X$ has a bottom element $\perp=\bigwedge X$ and a top element $\top=\bigvee X$. These are respectively the unique minimal and maximal elements of $X$.

The meet and join operations satisfy various commutative, associative, and distributive laws, which we do not list here. A complete lattice can be characterised using only the meet (or, dually, the join), and this result will be useful below. This is [3, theorem 2.16]:

THEOREM 4.1. Let X be a non-empty partially ordered set. Then the following properties are equivalent:

(a) $X$ is a complete lattice;

(b) $\bigwedge Y$ exists for all $Y \subseteq X$;

(c) $X$ has a top element $\top$ and $\bigwedge Y$ exists for all $Y \subseteq X$ with $Y \neq \varnothing$.

In fact, the join can then be defined by

$$
\bigvee X=\bigwedge U_{X}
$$

where $U_{X}$ is the set of all upper bounds for $X$, observing that this set is non-empty since $\top$ exists.

[3, corollary 2.17 of theorem $2 \cdot 16]$ states:

COROLlaRY 4.2. Let $S$ be a set and let $X$ be a family of subsets of $X$, partially ordered by inclusion, such that:

(a) $\cap Y \in X$ for every non-empty family of subsets $Y$ of $S$;

(b) $S \in X$.

Then $X$ is a complete lattice in which

$$
\bigwedge Y=\bigcap Y \quad \bigvee Y=\bigcap\{A \in X: \bigcup A \subseteq Y\} .
$$

A non-empty family of subsets of $S$ satisfying (a) is called an intersection structure. If (b) holds, the family is said to be topped. So Corollary 4.2 states that every topped intersection structure is a complete lattice.

The set of all equivalence relations on $S$ (viewed as subsets of $S \times S$ ) is easily seen to be a topped intersection structure ([3, example 2.19(2)]). Corollary 4.2 implies that this set is a complete lattice. Here the partial ordering is $\subseteq$.

Now suppose that $\mathcal{G}$ is a coupled cell network with cells $\mathcal{C}$ and a choice of total phase space $P$. Let $\mathrm{M}_{\mathcal{G}}$ be the complete lattice of all equivalence relations on $\mathcal{C}$. Associated with each 

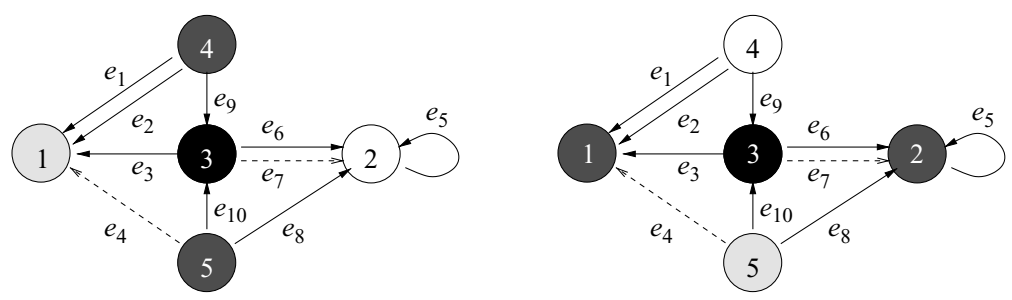

Fig. 3. Colourings of the network of Figure 1. (Left) Balanced. (Right) Unbalanced.

equivalence relation $\bowtie \in \mathrm{M}_{\mathcal{G}}$ is a subspace $\Delta_{\bowtie} \subseteq P$, called the polydiagonal corresponding to $\bowtie$. It is defined by

$$
\Delta_{\bowtie}=\left\{x \in P: c, d \in \mathcal{C} \text { and } c \bowtie d \Longrightarrow x_{c}=x_{d}\right\} .
$$

It consists of all $x$ whose components are equal for $\bowtie$-equivalent coordinates.

Define $\mathrm{W}_{\mathcal{G}}^{P}$ to be the set of all polydiagonals for this choice of $P$ and $\mathcal{G}$. There is a natural map

$$
\delta: \mathrm{M}_{\mathcal{G}} \longrightarrow \mathrm{W}_{\mathcal{G}}^{P} \quad \delta(\bowtie)=\Delta_{\bowtie}
$$

and it is easy to verify that $\delta$ is a bijection. It is also clear that the set $\mathrm{W}_{\mathcal{C}}^{P}$ is a topped intersection structure, hence a complete lattice.

LEMMA 4.3. The map $\delta$ is a lattice anti-isomorphism, that is, an isomorphism that reverses order, hence interchanges meet and join.

Recall ([3, section 1.17]) that the dual of a partially ordered set $S$ is the partially ordered set $S^{\partial}$ with the same elements as $S$, but where $x \leqslant y$ in $S^{\partial}$ if and only if $y \leqslant x$ in $S$. That is, the ordering is reversed. If we replace $\mathbf{M}_{\mathcal{G}}$ by its dual $\mathbf{M}_{\mathcal{G}}^{\partial}$ then we obtain a lattice isomorphism

$$
\delta^{\partial}: \mathrm{M}_{\mathcal{G}}^{\partial} \longrightarrow \mathrm{W}_{\mathcal{G}}^{P} \text {. }
$$

\section{Lattice of balanced equivalence relations}

In this section we prove that the balanced equivalence relations on a coupled cell network have a natural structure of a complete lattice. However, this lattice is not a sublattice of $\mathbf{M}_{\mathcal{G}}$.

\subsection{Balanced equivalence relations}

An equivalence relation $\bowtie$ on $\mathcal{C}$ determines a unique partition of $\mathcal{C}$ into $\bowtie$-equivalence classes, which can be interpreted as a colouring of $\mathcal{C}$ in which $\bowtie$-equivalent cells receive the same colour. Conversely, any partition (colouring) determines a unique equivalence relation.

Definition 5.1. An equivalence relation $\bowtie$ on $\mathcal{C}$ is balanced if for every $c, d \in \mathcal{C}$ with $c \bowtie d$, there exists $\beta \in B(c, d)$ such that $\mathcal{T}(i) \bowtie \mathcal{T}(\beta(i))$ for all $i \in I(c)$. The associated colouring is called a balanced colouring.

Remark 5.2. If $I(c)$ and $I(d)$ are empty, then the unique element in $B(c, d)=\{\varnothing\}$ is automatically a colour-preserving bijection, since that condition is vacuously true.

Example 5.3.

Consider the network in Figure 1. The colouring shown in Figure 3 (Left) is balanced by Remark 5.2. On the other hand the colouring in Figure 3 (Right) is not balanced, because $1 \bowtie 2$ but no input bijection $\beta: I(1) \rightarrow I(2)$ can preserve colour. Indeed $\mathcal{T}\left(e_{5}\right)=2$ has the same colour as cell 2 , but no arrow with head 1 has a tail cell of that colour. 
For future use we now state a combinatorial condition that is equivalent to the balance property. To do so, we need some notation.

Let $\mathcal{G}$ be a coupled cell network with cells $\mathcal{C}$. Let $c \in \mathcal{C}$ with input set $I(c)$. Let $A$ be an arrow-type (that is, a $\sim_{E}$-equivalence class of arrows) and let $K$ be a colour-type (that is, a $\bowtie$-equivalence class of cells). Define

$$
\mu_{A}(c, K)=|I(c) \cap A \cap K| .
$$

Further, define a subnetwork $\mathcal{G}_{A}$ to have the same cells as $\mathcal{G}$, but including only those edges that belong to $A$. The input set of $c$ in the network $\mathcal{G}_{A}$ is the same as our previously defined set $I^{A}(c)$.

[11, condition (6.2)] now leads directly to the following simple but useful result (which we prove in full for completeness):

LEMMA 5.4. Let $\mathcal{G}$ be a coupled cell network with cells $\mathcal{C}$, and let $\bowtie$ be an equivalence relation on $\mathcal{C}$. Then the following properties are equivalent:

(a) the relation $\bowtie$ is balanced;

(b) the restriction of $\bowtie$ to $\mathcal{G}_{A}$ is balanced for every arrow-type $A$;

(c) if $c, d \in \mathcal{C}$ and $c \bowtie d$ then

$$
\mu_{A}(c, K)=\mu_{A}(d, K)
$$

for all arrow-types $A$ and colour-types $K$.

Proof. Clearly (a) implies (b) and (b) implies (c). If (c) holds, then there exist bijections

$$
\beta_{A, K}: I^{A}(c) \cap K \longrightarrow I^{A}(d) \cap K
$$

for each arrow-type $A$ and colour-type $K$. Now define $\beta: I(c) \rightarrow I(d)$ by

$$
\beta(e)=\beta_{A(e), K(e)}(e)
$$

where $A(e)$ is the arrow-type containing $e$ and $K(e)$ is the colour-type of the tail cell $\mathcal{T}(e)$. Clearly $\beta$ is a $\bowtie$-preserving bijection, so $\bowtie$ is balanced and (a) holds.

If $\bowtie_{1}$ and $\bowtie_{2}$ are equivalence relations on a set $S$, then it is easy to verify that their intersection $\bowtie_{1} \cap \bowtie_{2}$ is also an equivalence relation. (Here we interpret the relations $\bowtie_{j}$ as subsets of $S \times S$.) This whole paper would be much simpler if the intersection of two balanced equivalence relations were always balanced. We now give an example to show that this property is false.

\section{Example 5.5.}

Figures 4 (Left) and 4 (Middle) show two colourings $\bowtie_{1}$ and $\bowtie_{2}$ of an 8-cell network in which every cell receives exactly two inputs, all cells are cell-equivalent, and all arrows are edge-equivalent. We claim that both colourings are balanced. Indeed, a case-by-case examination shows that in Figure 4 (Left) every black cell receives inputs from one black cell and one grey cell, and every grey cell receives inputs from one black cell and one grey cell. Similarly, in Figure 4 (Middle), every black cell receives inputs from one black cell and one grey cell, and every grey cell receives inputs from one black cell and one grey cell.

The colouring corresponding to the intersection of the above two equivalence relations is shown in Figure 4 (Right). Here two cells receive the same colour if and only if they do so for each of $\bowtie_{1}$ and $\bowtie_{2}$. So the corresponding equivalence relation is indeed $\bowtie_{1} \cap \bowtie_{2}$. 

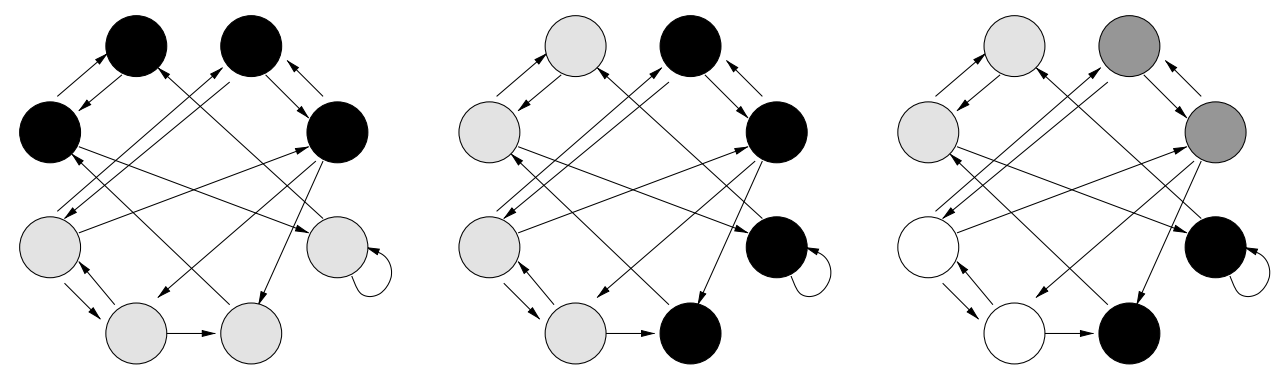

Fig. 4. The intersection (Right) of two balanced equivalence relations (Left, Middle) need not be balanced.

However, One black cell receives inputs from a white cell and a dark grey cell, whereas the other black cell receives inputs from a black cell and a pale grey cell. So this colouring is not balanced.

In view of the above example, we approach the latice of balanced equivalence relations indirectly, via the corresponding balanced polydiagonals. Specifically, we saw above that each equivalence relation $\bowtie$ on $\mathcal{C}$ defines a unique polydiagonal $\Delta_{\bowtie}$. If $\bowtie$ is balanced, we call $\Delta_{\bowtie}$ a balanced polydiagonal. We let $\Lambda_{\mathcal{G}}$ be the set of all balanced equivalence relations for $\mathcal{G}$, and denote the set of all balanced polydiagonals for $\mathcal{G}$ by $\mathrm{V}_{\mathcal{G}}^{P}$. Then

$$
\Lambda_{\mathcal{G}} \subseteq \mathrm{M}_{\mathcal{G}} \quad \mathrm{V}_{\mathcal{G}}^{P} \subseteq \mathrm{W}_{\mathcal{G}}^{P}
$$

We claim that $\Lambda_{\mathcal{G}}$ is a complete sublattice of $\mathbf{M}_{\mathcal{G}}$. Invoking the anti-isomorphism $\delta$, it suffices to prove that $\mathrm{V}_{\mathcal{G}}^{P}$ is a complete sublattice of $\mathrm{W}_{\mathcal{G}}^{P}$. We do this using admissible maps. To do this, say that a subspace $V$ of $P$ is admissibly invariant if $f(V) \subseteq V$ for every admissible map $f$ on $P$. A crucial property of a balanced equivalence relation is that it defines an admissibly invariant polydiagonal, and conversely:

THEOREM 5.6. Let $\bowtie$ be an equivalence relation on a coupled cell network. Then $\Delta_{\bowtie}$ is admissibly invariant if and only if $\bowtie$ is balanced.

Proof. A proof for finite networks is given in $[6, \mathbf{6 1}]$, in terms of admissible smooth vector fields and flow-invariant subspaces. It is obvious that balance implies admissible invariance. The converse uses only linear maps defined on finite sets of variables. We now show that this proof can be modified to work for networks of finite type.

Suppose that $\bowtie$ is an equivalence relation on $\mathcal{C}$ and that $\Delta_{\bowtie}$ is admissibly invariant. Then in particular it is invariant under all linear admissibly invariant maps. These are characterised in Theorem 3.7 in terms of the vectors $\bar{x}^{A, c}$ for cells $c$ and arrow-types $A$.

Choose a fixed but arbitrary $c \in \mathcal{C}$, and let $A$ be a fixed arrow-type. Choose a nonzero element $u \in P_{c}$, and define an element $v \in P$ by

$$
v_{j}=\left\{\begin{array}{lll}
u & \text { if } & j \sim_{I} c \\
0 & \text { if } & j+{ }_{I} c .
\end{array}\right.
$$

By construction, $v \in \Delta_{\bowtie \text {. }}$

Define a linear admissible map $f: P \rightarrow P$ by

$$
\left\{\begin{array}{l}
f_{c}(x)=\bar{x}^{A, c} \\
f_{d}(x)=\bar{\beta}^{*} x{ }^{A, c} \text { if } c \sim_{I} d \text { for } \beta \in B(c, d) \\
f_{c}(x)=0 \text { otherwise. }
\end{array}\right.
$$


Let $s=\left|\mathbf{S}_{n_{a}(c)}\right|$. Then $v \in \Delta_{\bowtie}$, and an easy calculation shows that if $K$ is the $\bowtie$ equivalence class containing $c$ then

$$
f_{d}(v)=\frac{\mu_{A}(d, K)}{s} u
$$

for all $d \in \mathcal{C}$ with $d \bowtie c$. (Note that $K$ is then the $\bowtie$-equivalence class containing $d$.)

Since we are assuming that $\bowtie$ is balanced, we must have $f(v) \in \Delta_{\bowtie}$. This is the case if and only if $f_{d}(v)=f_{c}(v)$ for all $d \sim_{I} c$. By (5.2) this is equivalent to $\mu_{A}(c, K)=\mu_{A}(d, K)$, and since this holds for all $c, A, K$ and all $d \bowtie c$, Lemma 5.4 completes the proof.

THEOREM 5.7. With the above notation, the sets $\Lambda_{\mathcal{G}}$ of balanced equivalence relations and $V_{\mathcal{G}}^{P}$ of balanced polydiagonals for $\mathcal{G}$ are complete lattices. They are canonically antiisomorphic.

Proof. It is obvious that the set of admissibly invariant maps forms a topped intersection structure. Therefore it is a complete lattice by the discussion immediately following Corollary 4.2. Now apply $\delta$.

\subsection{Lattice structure}

We now relate the lattice operations in $\Lambda_{\mathcal{G}}$ to a natural partial order on equivalence relations.

Let $\mathcal{G}$ be a coupled cell network with cells $\mathcal{C}$ and arrows $\mathcal{E}$. Let $\Lambda_{\mathcal{G}}$ be the set of all balanced equivalence relations on $\mathcal{C}$, and let $\bowtie_{1}, \bowtie_{2} \in \Lambda_{\mathcal{G}}$. We say that $\bowtie_{1}$ refines $\bowtie_{2}$, denoted by $\bowtie_{1} \prec \bowtie_{2}$, if and only if

$$
c \bowtie_{1} d \Longrightarrow c \bowtie_{2} d .
$$

That is, the partition of $\mathcal{C}$ defined by $\bowtie_{1}$ is finer than that defined by $\bowtie_{2}$ in the sense that for any $c \in \mathcal{C}$

$$
[c]_{1} \subseteq[c]_{2}
$$

where $[c]_{j}$ is the $\bowtie_{j}$-equivalence class of $c$ for $j=1,2$. Observe that $\prec$ is a partial ordering on $\Lambda_{\mathcal{G}}$. Indeed it is the restriction to $\Lambda_{\mathcal{G}}$ of the partial ordering $\subseteq$ on the set $\mathrm{M}_{\mathcal{G}}$ of all equivalence relations on $\mathcal{C}$. However, $\Lambda_{\mathcal{G}}$ is not a sublattice of $\mathrm{M}_{\mathcal{G}}$.

As in Lemma 4.3, forming polydiagonals reverses order:

$$
\bowtie_{1} \prec \triangleleft_{2} \Longleftrightarrow \Delta_{\bowtie_{1}} \supseteq \Delta_{\bowtie_{2}}
$$

\section{Finest and coarsest balanced equivalence relations}

Trivially, the unique finest (bottom) element of $\Lambda_{\mathcal{G}}$ is the relation $=$ of equality. Note that $\Delta_{=}=P$, so the largest possible polydiagonal corresponds to the finest possible balanced equivalence relation, as we expect from (5.3).

\subsection{Coarsest balanced equivalence relation}

Less obviously, there also exists a unique coarsest (top) balanced equivalence relation, where $\bowtie_{1}$ is coarser than $\bowtie_{2}$ if and only if $\bowtie_{2}$ is finer than $\bowtie_{1}$. The simplest way to prove this seems to be to use the connection between balanced equivalence relations and admissibly invariant maps.

The polydiagonal corresponding to the coarsest balanced equivalence relation must be the smallest balanced polydiagonal; that is, the intersection $I$ of all $\Delta_{\bowtie}$ over all balanced equivalence relations $\bowtie$. This intersection is non-trivial (that is, the set of balanced equivalence 
relations is non-empty) because equality is a balanced equivalence relation. It is easy to see that any intersection of polydiagonals is a polydiagonal. Moreover, the space $I$ is admissibly invariant, because each $\Delta_{\bowtie}$ is admissibly invariant. Therefore $I$ must correspond to some balanced equivalence relation, which we denote by $\bowtie \ll$.

The relation $\triangle \triangleleft \Delta$ refines input equivalence $\sim_{I}$, but need not equal $\sim_{I}$ because $\sim_{I}$ need not be balanced. Indeed, if $G$ is a chain of three identical cells $1 \leftarrow 2 \leftarrow 3$ where the arrows are identical, then the $\sim_{I}$-equivalence classes are $\{1,2\},\{3\}$. However, the equivalence relation $\sim_{I}$ is not balanced, because the input sets of cells 1 and 2 are $\{1,2\}$ and $\{2,3\}$ respectively, and $2+{ }_{I} 3$.

Since $\Lambda_{\mathcal{G}}$ is anti-isomorphic to $V_{\mathcal{G}}$, the meet and join of two balanced equivalence relations satisfy the following conditions:

$$
\begin{aligned}
& \Delta_{\bowtie_{1} \vee \bowtie_{2}}=\Delta_{\bowtie_{1}} \cap \Delta_{\bowtie_{2}} \\
& \Delta_{\bowtie_{1} \wedge \bowtie_{2}}=\Delta_{\varpi_{1}} \cup \Delta_{\triangleright_{2}} .
\end{aligned}
$$

It is straightforward to verify from the corresponding polydiagonals that $\bowtie_{1} \vee \bowtie_{2}$ equivalence can be characterised as follows:

PROPOSITION 6.1. Let $\bowtie_{3}=\bowtie_{1} \vee \bowtie_{2}$. Then $c \bowtie_{3} d$ if and only if there exists a finite chain

$$
c=c_{1}, \ldots, c_{k}=d
$$

such that for all $j$ with $1 \leqslant j \leqslant k-1$ either $c_{j} \bowtie_{1} c_{j+1}$ or $c_{j} \bowtie_{2} c_{j+1}$.

Equivalently, $\bowtie_{3}$ is the finest balanced equivalence relation such that

$$
c \bowtie_{1} d \quad \text { or } c \bowtie_{2} d \Longrightarrow c \bowtie_{3} d .
$$

We omit the proof, but observe that the same property characterises the join operation in the lattice of all equivalence relations.

A similar combinatorial characterisation of $\bowtie_{1} \wedge \bowtie_{2}$ seems more elusive. Example 5.5 shows that it is not the intersection $\bowtie_{1} \cap \bowtie_{2}$.

Remark 6.2. We have shown that the lattice of balanced equivalence relations is not a sublattice of the lattice of all equivalence relations, even though the join operation on equivalence relations restricts to give the join operation on balanced equivalence relations-and, moreover, the meet can be defined in terms of the join. It may be worth noting that this type of behaviour is fairly common. For example, consider the lattice of subsets of a finite group, and the lattice of subgroups of that group. In both cases the meet operation is intersection, but the join operation for subsets is the union, whereas for subgroups it is 'generated by'.

\subsection{Quotients}

Any balanced colouring $\bowtie$ on a network $\mathcal{G}$ determines a quotient network $\mathcal{G}_{\bowtie}$ in which all cells of any given colour are identified, while input set topology is preserved. The dynamics of $\mathcal{G}_{\bowtie}$ corresponds to synchronous dynamics of $\mathcal{G}$; that is, dynamics restricted to $\Delta_{\bowtie}$. See [6, 11].

Let $\Lambda_{\mathcal{G}}^{\bowtie}$ be the sublattice of $\Lambda_{\mathcal{G}}$ consisting of all balanced equivalence relations $\bowtie_{1}$ for which $\bowtie_{1} \prec \bowtie$. The following is straightforward.

PROPOSITION 6.3. There is a natural lattice isomorphism between $\Lambda_{\mathcal{G} / \bowtie}$ and $\Lambda_{\mathcal{G}}^{凶}$.

Proof. The proof is routine but tedious and will be omitted. 

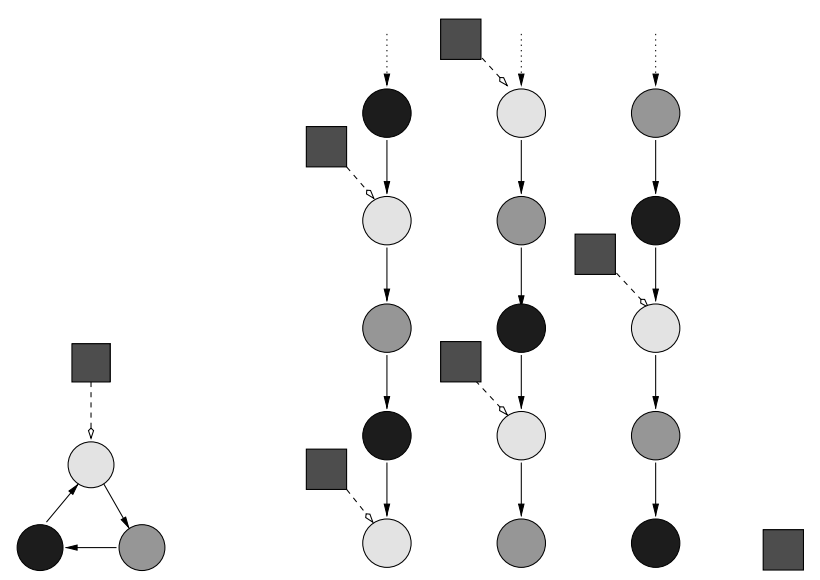

Fig. 5. (Left) a network. (Right) its universal cover.

\section{Paths, coverings and the coarsest Relation}

We seek to characterise the top element of $\Lambda_{\mathcal{G}}$. To do so, we employ the universal cover $U(\mathcal{G})$ of the graph $\mathcal{G}$, defined as follows. The nodes of $U(\mathcal{G})$ are all the finite directed paths in $\mathcal{G}$, that is, finite sequences of edges $\left(e_{1}, e_{2}, \ldots, e_{k}\right)$ such that the tail of $e_{i}$ is equal to the head of $e_{i+1}$ for $1 \leqslant i \leqslant k-1$. Schematically,

$$
e_{1} \longleftarrow e_{2} \longleftarrow e_{3} \longleftarrow \cdots \longleftarrow e_{k}
$$

where

$$
e \longleftarrow e^{\prime} \Longleftrightarrow t(e)=h\left(e^{\prime}\right)
$$

(We allow trivial paths with $k=0$ and identify these with individual nodes.)

For each such path we introduce an arrow from node $\left(e_{1}, e_{2}, \ldots, e_{k}\right)$ to $\left(e_{1}, e_{2}, \ldots, e_{k-1}\right)$ of the same type as the arrow from $e_{k}$ to $e_{k-1}$ in the path $\left(e_{1}, e_{2}, \ldots, e_{k}\right)$. There is a canonical projection $\pi^{U}$ from $U(\mathcal{G})$ to $\mathcal{G}$, the covering map, which sends node $\left(e_{1}, e_{2}, \ldots, e_{k}\right)$ to the head of $e_{1}$, and maps arrows according to the above definition. The covering map $\pi^{U}$ is a quotient map. When $\mathcal{G}$ is finite, the graph $U(\mathcal{G})$ is finite if $\mathcal{G}$ contains no closed directed paths, and is infinite otherwise.

\section{Example 7.1.}

Suppose that $\mathcal{G}$ is the 4-cell network of Figure 5 (Left). Then its universal cover $U(\mathcal{G})$ is shown in Figure 5 (Right). Observe that $U(\mathcal{G})$ has four connected components, each of which is a directed tree. So $U(\mathcal{G})$ is a forest, Wilson [12].

Definition 7.2. The input tree of a node $c \in \mathcal{C}$ is the subgraph $I^{\infty}(c)=\left(\pi^{U}\right)^{-1}(c)$ of $U(\mathcal{G})$. It consists of all paths that terminate in $c$.

The terminology reflects the obvious fact that $I^{\infty}(c)$ is a directed tree, with root $c$. If $\mathcal{G}$ has multiple arrows, these are separated into arrows of multiplicity 1 in $U(\mathcal{G})$ and hence in any $I^{\infty}(c)$.

Similarly we define the $k$ th iterated input set $I^{k}(c)$ to be the subtree of $I^{\infty}(c)$ whose nodes are all paths of length $k$ that terminate at $c$. Clearly

$$
I^{\infty}(c)=\bigcup_{k=0}^{\infty} I^{k}(c) .
$$




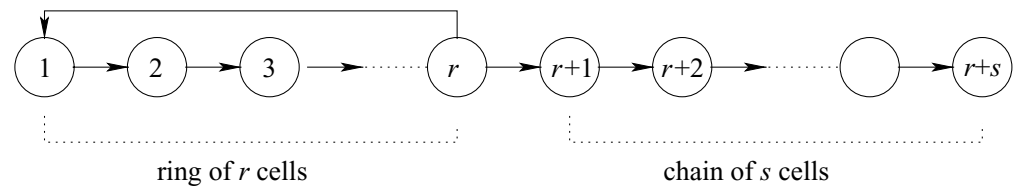

Fig. 6. Chain with feedback loop.

THEOREM 7-3. Let $\mathcal{G}$ be a coupled cell network on a set of cells $\mathcal{C}$, and let $c, c^{\prime} \in \mathcal{C}$. Then $c \infty d$ in $\mathcal{G}$ if and only if $I^{\infty}\left(c^{\prime}\right)$ and $I^{\infty}\left(d^{\prime}\right)$ are isomorphic.

Proof. Define an equivalence relation $\sim_{T}$ by:

$$
c \sim_{T} c^{\prime} \Longleftrightarrow I^{\infty}(c) \cong I^{\infty}\left(c^{\prime}\right) .
$$

We claim that $\sim_{T}$ is balanced, and that each of $\sim_{T}$ and $\bowtie$ refines the other. These statements prove that the two relations are equal.

(1) The relation $\sim_{T}$ is balanced.

Suppose that $c \sim_{T} c^{\prime}$. Then there is an isomorphism $\psi: I^{\infty}(c) \rightarrow I^{\infty}\left(c^{\prime}\right)$. By definition, $\psi$ preserves the root of the tree, so $\psi(c)=c^{\prime}$. By induction on $k$ it is clear that $\psi\left(I^{k}(c)\right)=$ $I^{k}\left(c^{\prime}\right)$. Therefore $\psi\left(I^{\infty}(c)\right)=I^{\infty}\left(c^{\prime}\right)$, on taking the obvious direct limit. Since $I^{\infty}(c)$ and $I^{\infty}\left(c^{\prime}\right)$ are directed trees, we deduce that for each $i \in I(c)$ and for all $k \geqslant 0$ we have $\psi\left(I^{k}(i)\right)=I^{k}(\psi(i))$, so $\psi\left(I^{\infty}(i)\right)=I^{\infty}(\psi(i))$, and $i \sim_{T} \psi(i)$. Therefore $\psi$ preserves $\sim_{T}$ so the relation $\sim_{T}$ is balanced.

(2) $\sim_{T}$ refines $\bowtie$.

This is immediate from part 1 since any balanced equivalence relation refines

(3) $\bowtie$ refines $\sim_{T}$.

More generally, any balanced equivalence relation $\bowtie$ refines $\sim_{T}$. For suppose that $c \bowtie c^{\prime}$. Then there is an input isomorphism $\beta: I(c) \rightarrow I\left(c^{\prime}\right)$. Moreover, for any $i \in I(c)$ we have $\beta(i) \bowtie i$. Inductively on $k$ we see that there exists an isomorphism of graphs

$$
\beta^{k}: I^{k}(c) \longrightarrow I^{k}(\beta(c))
$$

such that $\beta^{1}=\beta$ and $\beta^{k}$ extends $\beta^{k-1}$. Define $\beta^{\infty}=\bigcup_{k=1}^{\infty} \beta^{k}$. Then $\beta^{\infty}: I^{\infty}(c) \rightarrow I^{\infty}\left(c^{\prime}\right)$ is an isomorphism. Therefore $c \sim_{T} c^{\prime}$ and we are done.

This theorem characterises in terms of an infinite construction. Aldis [1] describes a polynomial-time algorithm to compute truncate $I^{\infty}(c)$ to some finite tree $I^{k}(c)$ for suitably large $k$. However, the algorithm has other features to enhance its efficiency.

\section{Chains with feedback}

As an example, we now discuss the lattice $\Lambda_{\mathcal{G}}$ when $\mathcal{G}=G_{r s}$ is a chain with feedback as shown in Figure 6.

We introduce two standard lattices. If $n \in \mathbf{N}$ we define the lattice $C_{n}$ to consist of the elements $0,1,2, \cdots, n$ in their natural order. This is a chain of length $n+1$.

If further $n>0$ we define $D_{n}$ to be the lattice of all divisors of $n$, with $d \prec d^{\prime}$ if and only if $d \mid d^{\prime}$.

We now have:

PROPOSITION 8.1. The lattice $\Lambda_{G_{r s}}$ is isomorphic to the direct product $D_{r} \times C_{s}$. 
Proof. Let $\bowtie$ be a balanced equivalence relation on $G_{r s}$. Work backwards from cell $r+s$ until reaching the first cell $j$ such that $j \bowtie j^{\prime}$ with $j^{\prime}>j$.

If no such cell exists, then $\bowtie$ is equality.

Otherwise, the sequence of equivalence classes (colours) from $j^{\prime}$ to $j+1$ must repeat periodically for all cells with number $\leqslant j^{\prime}$, because of the balance condition $(\mathcal{T}(I(c))=$ $\{c-1\})$. Let $d$ be the length of this minimal repeating block. For consistency at cell $r$ we must have $d \mid r$. Moreover, the entire ring must be filled with this periodic sequence.

We claim that there are no further conditions on $\bowtie$. Specifically, we can construct a balanced equivalence relation $\bowtie_{k a}$ for any divisor $k \mid r$ and $0 \leqslant a \leqslant s$ as follows. Repeat a sequence of $k$ distinct colours round the ring. Continue this sequence up to cell $r+t$ with $0 \leqslant t \leqslant s$, where $t=s-a$. Finish by making the remaining cells $r+t+1, \ldots, r+s$ of different colours that are not in the periodic sequence.

It is easy to check that the equivalence relation $\bowtie_{k a}$ is balanced, and that every balanced equivalence relation found above (including equality) is of the form $\bowtie_{k a}$.

Therefore $\Lambda_{G_{r s}}$ consists of the relations $\bowtie_{k a}$ for $k \mid r$ and $0 \leqslant a \leqslant s$. To find the ordering $\prec$, verify (which is routine) that

$$
\bowtie_{k^{\prime} a^{\prime}} \prec \bowtie_{k a} \Longleftrightarrow k \mid k^{\prime} \text { and } a \leqslant a^{\prime} .
$$

Therefore

$$
\Lambda_{G_{r s}} \cong\left(D_{r} \times C_{s}\right)^{\partial} .
$$

But $\left(D_{r} \times C_{s}\right)^{\partial} \equiv D_{r}^{\partial} \times C_{s}^{\partial} \equiv D_{r}^{\times} C_{s}$ since both $D_{r}$ and $C_{s}$ are self-dual, as claimed.

COROLLARY 8.2. The lattice $\Lambda_{G_{r s}}$ contains $(s+1) d(r)$ elements, where $d(r)$ is the number of divisors of $r$.

It is possible for a network to have no balanced equivalence relations except equality. An example is a linear chain of $n$ identical cells without feedback.

At the other extreme is the complete graph $G=K_{n}$ on $n$ cells, where every cell is connected to every other cell and all edges are identical. The network $K_{n}$ is homogeneous, and any equivalence relation on $\{1, \ldots, n\}$ is balanced. Since equivalence relations correspond bijectively to partitions, the number of elements is

$$
\left|\Lambda_{K_{n}}\right|=\mathbf{B}_{n}
$$

where $\mathbf{B}_{n}$ is the $n$th Bell number, see Graham et al. [7, page 373, exercise 15]. This is defined by

$$
\mathbf{B}_{n}=\sum_{k=0}^{n}\left\{\begin{array}{l}
n \\
k
\end{array}\right\}
$$

where the braces denote Stirling numbers of the second kind [7, page 258].

Acknowledgements. This research was partially supported by a grant from EPSRC.

\section{REFERENCES}

[1] J. ALDIS. A polynomial time algorithm to determine maximal balanced equivalence relations. Internat J. Bifur Chaos Appl. Sci. Engrg., to appear.

[2] F. ANTONELi AND I. STEwART. Symmetry and synchrony in coupled cell networks 2: group networks. Internat. J. Bifur Chaos Appl. Sci. Engrg., to appear.

[3] B. A. Davey And H. A. PRiestley. Introduction to Lattices and Order (Cambridge University Press, 1990). 
[4] W. Fulton And J. Harris. Representation Theory. Graduate Texts in Math. 129 (Springer, 1991).

[5] M. Golubitsky AND I. STEWART. Nonlinear dynamics of networks: the groupoid formalism. Bull. Amer. Math. Soc. 43(2006), 305-364.

[6] M. Golubitsky, I. STEwART AND A. TÖRÖK. Patterns of synchrony in coupled cell networks with multiple arrows. SIAM J. Appl. Dynam. Sys. 4(1) (2005), 78-100.

[7] R. L. Graham, D. E. Knuth and O. PatashniK. Concrete Mathematics (Addison-Wesley, 1994).

[8] P. J. Higgins. Notes on Categories and Groupoids. Van Nostrand Reinhold Mathematical Studies 32 (Van Nostrand Reinhold, 1971).

[9] S. C. Manrubia, A. S. Mikhailov and D. H. Zanette. Emergence of Dynamical Order (World Scientific, 2004).

[10] E. Mosekilde, Y. Maistrenko and D. Postonov. Chaotic Synchronization (World Scientific, 2002).

[11] I. Stewart, M. Golubitsky and M. Pivato. Patterns of synchrony in coupled cell networks. SIAM J. Appl. Dynam. Sys 2 (2003), 609-646. [DOI: 10.1137/S1111111103419896].

[12] R. J. WILSON. Introduction to Graph Theory (3rd ed.) (Longman, 1985).

[13] C. W. Wu. Synchronization in Coupled Chaotic Circuits and Systems (World Scientific, 2002).

[14] C. W. WU. Synchronization in networks of nonlinear dynamical systems coupled via a directed graph. Nonlinearity 18 (2005), 1057-1064. 